\title{
Conjunctival Intraepithelial Neoplasia with Mucoepidermoid Differentiation: A Case Report of a Subtle Lesion
}

\author{
Madhura G. Joag a Anita Gupta ${ }^{a} \quad$ Anat Galora, b Sander R. Dubovy a, c \\ Jose Antonio Bermudez-Magner ${ }^{a}$ c Jianhua Wang ${ }^{a}$ Carol L. Karp ${ }^{a}$ \\ a Bascom Palmer Eye Institute, University of Miami, b Miami Veterans Affairs Medical Center, \\ and 'Florida Lions Eye Bank, Ocular Pathology Laboratory, Miami, Fla., USA
}

\section{Key Words}

Conjunctival intraepithelial neoplasia $\cdot$ Mucoepidermoid differentiation $\cdot$ Neoplasia $\cdot$ Optical coherence tomography · Imaging

\begin{abstract}
Objective: To describe the clinical presentation, diagnostic imaging, and treatment options of conjunctival intraepithelial neoplasia (CIN) with mucoepidermoid differentiation, an in situ stage of mucoepidermoid carcinoma of the conjunctiva (MCC). Results: We report the case of an 86-year-old man presenting with a subtle limbal lesion that had only mild erythema and elevation. Based on a few atypical clinical features and an abnormal ultrahigh-resolution optical coherence tomography (UHR OCT), an incisional biopsy was performed revealing CIN with mucoepidermoid differentiation. Treatment involved aggressive surgical excision. No evidence of recurrence was noted in the 5 years of follow-up. Conclusion: MCC is an aggressive tumor that has a tendency to be invasive and recur after treatment. Therefore, early diagnosis and treatment is critical. CIN with mucoepidermoid differentiation may represent the earliest stage of MCC. It can present subtly, with a clinical resemblance to benign and less aggressive ocular surface lesions. Imaging with UHR OCT may be helpful to detect early neoplasia. A high level of suspicion must be maintained when evaluating potential ocular surface tumors.
\end{abstract}

(C) 2015 S. Karger AG, Base

\section{Introduction}

Mucoepidermoid carcinoma of the conjunctiva (MCC) is a rare tumor affecting the ocular surface [1] and is a variant of squamous cell carcinoma (SCC). It is typically aggressive, with a higher propensity than SCC for local invasion and recurrence after treatment [2,3]. For this 
Ocular Oncology

and Pathology

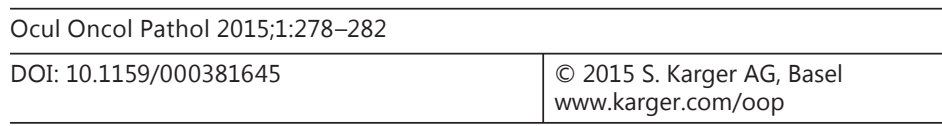

Joag et al.: Conjunctival Intraepithelial Neoplasia with Mucoepidermoid Differentiation: A Case Report of a Subtle Lesion

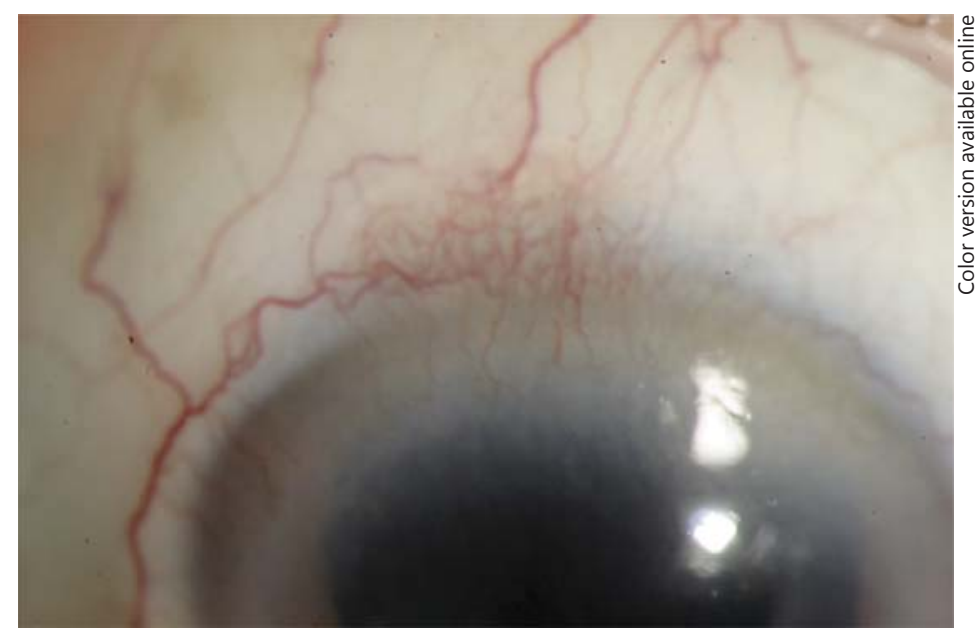

Fig. 1. Subtle clinical presentation of CIN. Only mild superior and superior temporal limbal inflammation and pannus are seen.

reason, early diagnosis is critical in minimizing morbidity. We report a unique subtle case of conjunctival intraepithelial neoplasia (CIN) that demonstrated mucoepidermoid differentiation. Such tumors have been described as an early and preinvasive stage of MCC [4]. In this case, imaging with ultrahigh-resolution optical coherence tomography (UHR OCT) was key in raising the clinical suspicion of neoplasia. This case was successfully managed with surgical excision, mitomycin $\mathrm{C}$, and cryotherapy.

\section{Case Report}

This is a case report of a patient who was diagnosed and treated at the Bascom Palmer Eye Institute for CIN with mucoepidermoid differentiation.

An 86-year-old man presented to the Bascom Palmer Eye Institute with foreign body sensation and redness of the right eye for 6 months. He denied any vision changes, discharge, or pruritis. His past ocular history was significant only for pseudophakia, and his medical history was noncontributory.

At presentation, his best-corrected visual acuity in the right eye was 20/30 with mild conjunctival injection of the superior-temporal limbus and an adjacent small area of pannus formation (fig. 1). The area was minimally elevated, and there was no staining with Rose Bengal. However, a large feeder vessel was noted, making it suspicious for a neoplastic lesion.

UHR OCT of the lesion was obtained. We used a custom-built spectral domain anterior segment UHR OCT which has a center wavelength of $840 \mathrm{~nm}$, allowing for imaging with an axial resolution of approximately $3 \mu \mathrm{m}$ [5]. UHR OCT showed marked thickening of the corneal epithelium in the area of the lesion, which is suggestive of proliferating epithelium seen in epithelial tumors such as corneal intraepithelial neoplasia and CIN [5, 6]. In addition, the lesion was dense and irregular, causing shadowing posterior to the lesion (fig. 2a). This can be contrasted with the normal cornea in which the epithelium is uniform and thin (fig. 2b).

With the UHR OCT suggesting possible malignancy, a conjunctival biopsy was performed, revealing fullthickness dysplasia (carcinoma in situ) with mucoepidermoid differentiation (fig. 3). The patient then underwent surgical excision of the tumor. The tumor was carefully resected using 5-mm margins, along with epitheliectomy of the corneal extent of the tumor. The tumor was not adherent to the underlying sclera; however, the sclera appeared irregular and hyperemic at the superior limbus, so a partial-thickness sclerectomy was performed. Mitomycin C $(0.2 \mathrm{mg} / \mathrm{ml})$ was applied to the scleral bed for $1 \mathrm{~min}$. Double-freeze slow-thaw cryotherapy was also applied to the conjunctival margins and limbus.

Histopathologic examination confirmed CIN with mucoepidermoid differentiation and with clear conjunctival margins. The epithelium displayed faulty epithelial maturational sequencing that extended up to full thickness (fig. 3a). The cells were variably pleomorphic with variably prominent nucleoli. Intercellular material was identified which was positive for mucicarmine (fig. 3c), colloidal iron, Alcian blue, periodic acid- 
Ocular Oncology

and Pathology
Joag et al.: Conjunctival Intraepithelial Neoplasia with Mucoepidermoid Differentiation: A Case Report of a Subtle Lesion
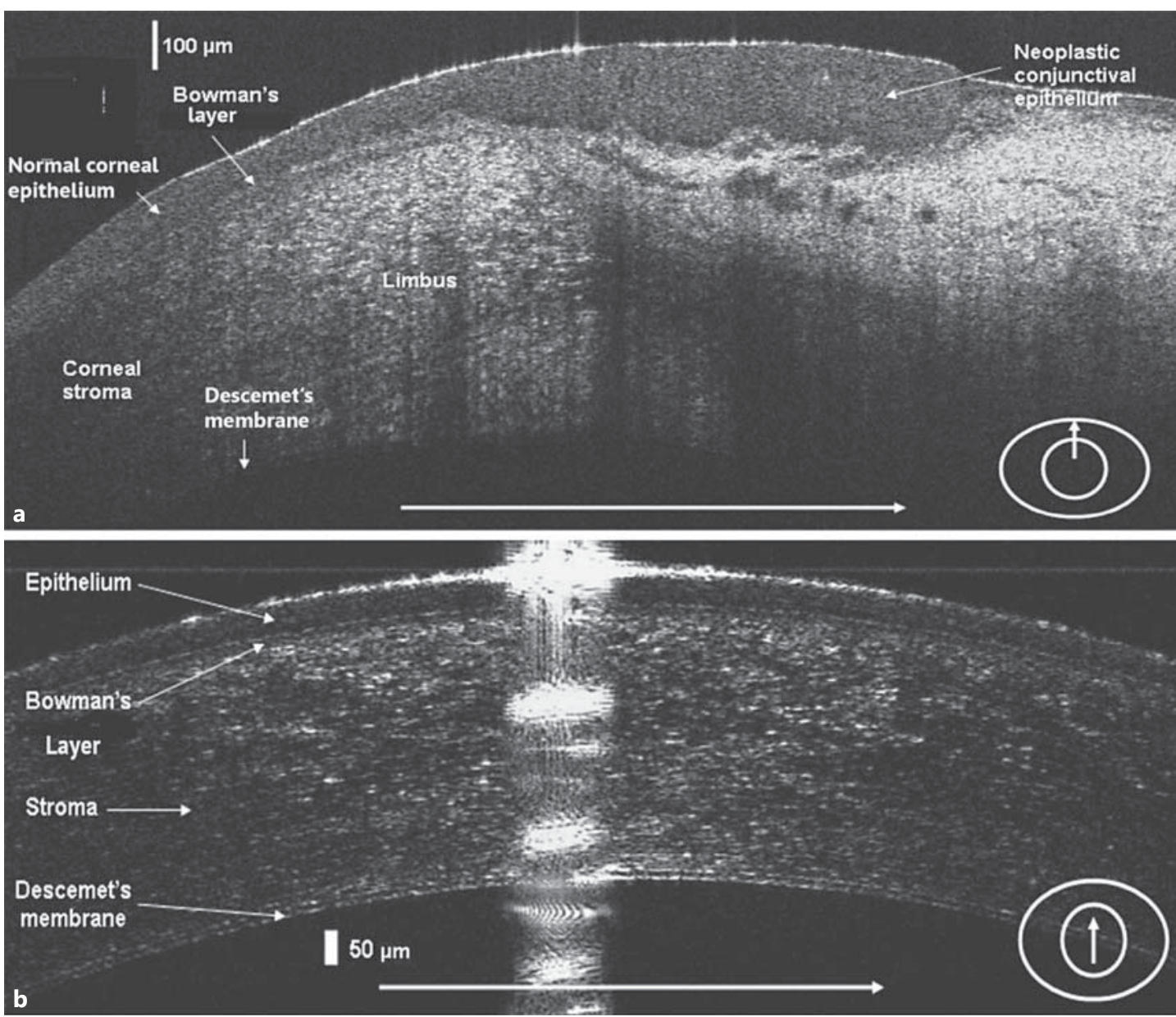

Fig. 2. a UHR OCT of intraepithelial neoplasia demonstrating marked thickening of the conjunctival epithelium. b UHR OCT of the normal cornea.

Schiff (PAS), and PAS with diastase stains (fig. 3b). Immunohistochemical stains for HMWK, Muc-1, CK, EMA, and CEA were all strongly positive. The Ki-67 stain (Mib-1) proliferation rate was approximately $20 \%$.

Sclerectomy tissue showed only chronic inflammatory cells, without tumor cells. The patient was treated postoperatively with topical fluoroquinolone for 1 week and a topical prednisolone taper for 1 month. No evidence of recurrence was noted at a follow-up period of 5 years.

\section{Discussion}

Mucoepidermoid carcinoma is a tumor that typically affects the major salivary glands and the upper respiratory tract; however, in rare instances it may also arise from the conjunctiva [1, 2, 7-11]. A review of 43 cases of MCC by Moloney et al. [12] revealed that this tumor generally occurs as a limbal lesion, around the 7th decade, with males more commonly affected than females. The disease course is typically rapid and aggressive, with a high recurrence rate after both simple excision (70\%) and wide local excision (44\%). The tumor has a high propensity for local recurrence and invasion. Exenteration/enucleation were required in $65 \%$ of the cases for local control of the tumor. 
Ocular Oncology
andPathology

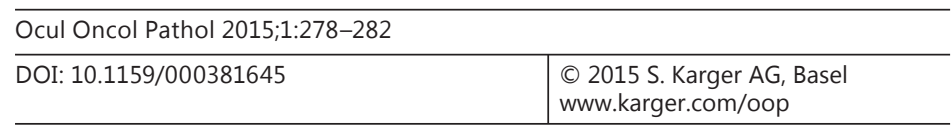

Joag et al.: Conjunctival Intraepithelial Neoplasia with Mucoepidermoid Differentiation: A Case Report of a Subtle Lesion

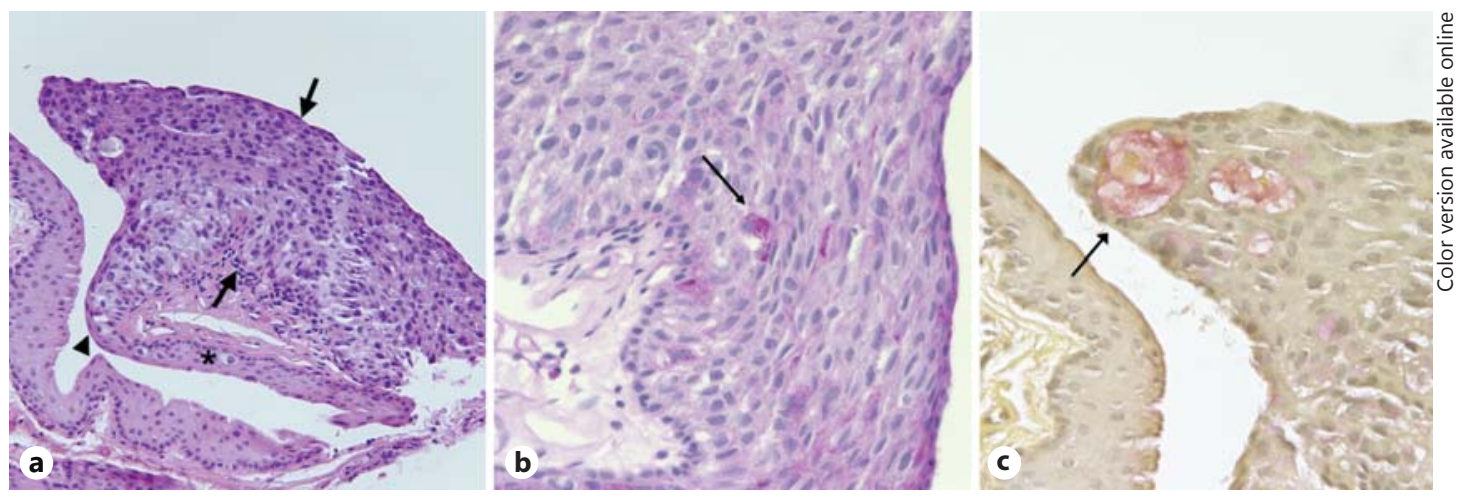

Fig. 3. a CIN with mucoepidermoid differentiation: conjunctiva that displays full-thickness faulty epithelial maturational sequencing (between arrows) transitions (arrowhead) to unremarkable conjunctiva (asterisk). HE. $\times 200$. b CIN with mucoepidermoid differentiation: diastase-resistant PAS-positive material is present within the area of full-thickness epithelial dysplasia (arrow). PAS. $\times 600$. c CIN with mucoepidermoid differentiation: mucinpositive material is present within the area of full thickness epithelial dysplasia (arrow). Mucicarmine. $\times 600$.

Although distant metastasis has not been reported, metastasis to local lymph nodes has been described [2]. CIN with mucoepidermoid differentiation is a potential precursor to MCC. For this reason, diagnosis of MCC at its earliest stages can be critical in reducing morbidity.

Diagnosis of CIN with mucoepidermoid differentiation can be made histopathologically by utilizing special stains for mucin (e.g. PAS, Alcian blue, or mucicarmine), which is present in dysplastic mucin-producing cells, but not in typical CIN [10]. Although the presence of mucin-producing cells in CIN suggests that the lesion has mucoepidermoid differentiation, the absence of mucin staining does not rule it out. Brownstein [7] reported a case of MCC where only the recurrent intraocular component of the tumor stained for mucin, while the primary lesion was histopathologically identical to SCC, lacking mucin-producing cells.

We report a case of CIN with mucoepidermoid differentiation in which the clinical presentation was subtle, as the lesion was only mildly elevated and lacked staining with Rose Bengal. The main suspicion for malignancy was the presence of a feeder vessel and the unilaterality of the lesion. In addition, imaging with UHR OCT was very helpful in suggesting an epithelial malignancy. UHR OCT is a promising technology for the noninvasive evaluation of patients with ocular surface lesions. The high-axial-resolution cross-sectional images of the ocular surface provides a detailed view of the epithelium. A thickened and usually hyperreflective epithelium is suggestive of epithelial proliferation seen in CIN. In this case, the patient demonstrated only mild clinical findings, but the UHR OCT led to our suspicion of epithelial neoplasia. Hopefully, future commercially available UHR OCT units will allow all clinicians to have this technology soon.

CIN with mucoepidermoid differentiation is a rare tumor, with only one other case previously reported in the English medical literature [4]. Given its potential as a precursor for the more aggressive MCC, appropriate treatment is essential. We treated our patient successfully with local excision using 5-mm margins, as well as adjuvant double-freeze slow-thaw cryotherapy, cautery, and mitomycin C $(0.2 \mathrm{mg} / \mathrm{ml})$ to the surgical bed in order to render any remaining tumor cells nonviable. Our patient showed no evidence of recurrence at 5 years of follow-up. Close and careful postsurgical monitoring for evidence of recurrence is also necessary in the management of CIN with mucoepidermoid differentiation. 
CIN with mucoepidermoid differentiation may represent the earliest stage of MCC. It can present subtly, with a clinical resemblance to benign and less aggressive ocular surface lesions. For this reason, a high level of suspicion must be maintained when evaluating potential ocular surface tumors.

\section{Acknowledgements}

This paper was financially supported by NIH Center Core Grant P30EY014801, RPB Unrestricted Award and Career Development Awards, Department of Defense (DOD grant No. W81XWH-09-1-0675), The Ronald and Alicia Lepke Grant, The Lee and Claire Hager Grant, The Jimmy and Gaye Bryan Grant, Richard Azar Family Grant, The Grant and Diana Thornbrough Grant and The Gordon Charitable Trust (all institutional grants).

\section{Statement of Ethics}

Written informed consent was obtained from the patient for the purpose of publication.

\section{Disclosure Statement}

The authors have no financial interest in any materials or methods presented in this paper.

\section{References}

1 Rao NA, Font RL: Mucoepidermoid carcinoma of the conjunctiva: a clinicopathologic study of five cases. Cancer 1976;38:1699-1709.

-2 Hwang IP, Jordan DR, Brownstein S, Gilberg SM, McEachren TM, Prokopetz R: Mucoepidermoid carcinoma of the conjunctiva: a series of three cases. Ophthalmology 2000;107:801-805.

3 Rankin JK, Jakobiec FA, Zakka FR, Foster CS: An improved approach to diagnosing and treating conjunctival mucoepidermoid carcinoma. Surv Ophthalmol 2012;57:337-346.

4 Margo CE, Groden LR: Intraepithelial neoplasia of the conjunctiva with mucoepidermoid differentiation. Am J Ophthalmol 1989;108:600-601.

5 Thomas BJ, Galor A, Nanji AA, El Sayyad F, Wang J, Dubovy SR, et al: Ultra high-resolution anterior segment optical coherence tomography in the diagnosis and management of ocular surface squamous neoplasia. Ocul Surf 2014;12:46-58.

-6 Shousha MA, Karp CL, Perez VL, Hoffmann R, Ventura R, Chang V, et al: Diagnosis and management of conjunctival and corneal intraepithelial neoplasia using ultra high-resolution optical coherence tomography. Ophthalmology 2011;118:1531-1537.

7 Brownstein S: Mucoepidermoid carcinoma of the conjunctiva with intraocular invasion. Ophthalmology 1981; 88:1226-1230.

-8 Carrau RL, Stillman E, Canaan RE: Mucoepidermoid carcinoma of the conjunctiva. Ophthal Plast Reconstr Surg 1994;10:163-168.

-9 Gamel JW, Eiferman RA, Guibor P: Mucoepidermoid carcinoma of the conjunctiva. Arch Ophthalmol 1984;102: 730-731.

10 Jastrzebski A, Brownstein S, Jordan DR, Gilberg SM: Histochemical analysis and immunohistochemical profile of mucoepidermoid carcinoma of the conjunctiva. Saudi J Ophthalmol 2012;26:205-210.

11 Robinson JW, Brownstein S, Jordan DR, Hodge WG: Conjunctival mucoepidermoid carcinoma in a patient with ocular cicatricial pemphigoid and a review of the literature. Surv Ophthalmol 2006;51:513-519.

12 Moloney TP, Trinh T, Farrah JJ: A case of conjunctival mucoepidermoid carcinoma in Australia. Clin Ophthalmol 2014;8:11-14. 